\title{
Diffusion Tensor Imaging of the Median Nerve: A Systematic Review and Meta-Analysis of Normal Values in Asymptomatic Adults and How They Change in Carpal Tunnel Syndrome
}

\section{Djamila Rojoa}

Leicester Royal Infirmary

Firas Raheman

Leicester Royal Infirmary

Joseph Rassam

Leicester Royal Infirmary

Ryckie George Wade ( $\sim$ ryckiewade@gmail.com )

University of Leeds

\section{Research Article}

Keywords: Diffusion tensor, normal, normative, healthy, asymptomatic, compressive neuropathy, peripheral nerve, median nerve, carpal tunnel syndrome, DTI, anisotropy

Posted Date: July 15th, 2021

DOI: https://doi.org/10.21203/rs.3.rs-697643/v1

License: (9) This work is licensed under a Creative Commons Attribution 4.0 International License.

Read Full License 


\section{Abstract}

Background: Carpal tunnel syndrome (CTS) leads to distortion of axonal architecture, demyelination and fibrosis within the median nerve. Diffusion tensor imaging (DTI) characterises tissue microstructure and generates reproducible proxy measures of nerve 'health' which are sensitive to myelination, axon diameter, fibre density and organisation. This meta-analysis summarises the normal DTI values of the median nerve, and how they change in CTS.

Methods: This systematic review included studies reporting DTI of the median nerve at the level of the wrist in adults. The primary outcome was to determine the normal fractional anisotropy (FA) and mean diffusivity (MD) of the median nerve. Secondarily, we show how the FA and MD differ between asymptomatic adults and patients with CTS, and how these differences are independent of the acquisition methods.

Results: 32 studies of 2643 wrists, belonging to 1575 asymptomatic adults and 1068 patients with CTS were included. The normal FA was $0.58(95 \% \mathrm{Cl} 0.56,0.59)$ and the normal MD was $1.138 \times 10^{-3} \mathrm{~mm}^{2} / \mathrm{s}$ $(95 \% \mathrm{Cl} 1 \cdot 101,1 \cdot 174)$. Patients with CTS had a significantly lower FA than controls (mean difference $0 \cdot 12$ $[95 \% \mathrm{Cl} 0.09,0.16])$. Similarly, the median nerve of patients with CTS had a significantly higher mean diffusivity (mean difference $0.16 \mathrm{~mm}^{2} / \mathrm{s} \times 10^{-3}[95 \% \mathrm{Cl} 0.05,0 \cdot 27]$ ). The differences were independent of experimental factors.

Conclusion: We provide summary estimates of the normal FA and MD of the median nerve in asymptomatic adults. Furthermore, we show that diffusion throughout the length of the median nerve becomes more isotropic in patients with CTS.

\section{Introduction}

Carpal tunnel syndrome (CTS) is the most common compressive neuropathy, affecting 10 million people annually. Consequently, CTS is the most expensive upper extremity musculoskeletal disorder, costing the USA health system over $\$ 2$ billion annually and employers up to $\$ 114,000$ per incident. ${ }^{1}$

Compression of peripheral nerves leads to distortion of the axonal architecture, demyelination with or without poor remyelination, loss of the intrinsic vasculature and ultimately, fibrosis of the perineurial and epineurial connective tissue. ${ }^{2,3}$ Diffusion tensor imaging (DTI) characterises tissue microstructure and generates reproducible ${ }^{4-7}$ proxy measures of nerve 'health' which are sensitive to myelination, axon diameter, fibre density and organisation ${ }^{8-10}$ (Figure 1). DTI typically generates the following metrics: fractional anisotropy (FA), mean diffusivity (MD), axial diffusivity (AD) and radial diffusivity (RD). FA is a scalar value between zero and one - an FA of zero implies isotropic diffusion of water within a voxel, whilst a FA of one implies diffusion along a single axis (i.e., bidirectional diffusion along the length of the nerve). MD describes the average molecular diffusion rate within the voxel, whilst $A D$ describes diffusion in the long axis and RD represents diffusion perpendicular to the long axis. 
Several studies have shown that DTI metrics (FA and MD) are sensitive to microstructural changes which occur within the median nerve of patients with CTS (Figure 1). However, there are several uncertainties must be resolved before this technology could be used in clinical practice or as a reference standard in research studies. Firstly, the normal DTI values of the median nerve must be established and secondly, uncertainty around how experimental conditions (e.g. scanning parameters) influence DTI metrics need to be determined. These uncertainties, and how DTI metrics change in CTS, might be resolved through metaanalysis and represents the rationale for this study.

\section{Methods}

This review is registered with PROSPERO (CRD42020212378). It was designed and conducted in accordance with the Cochrane Handbook of Systematic Reviews ${ }^{11}$ and has been authored in accordance with the PRISMA 2020 statement $^{12}$.

\section{Types of Studies}

We included all studies which reported the findings of diffusion tensor magnetic resonance imaging of the median nerve, at the level of the wrist in asymptomatic adults or adults with CTS. There were no language restrictions. We excluded case reports and studies which did not report DTI metrics (e.g., studies which contained fibre tractography graphics only) of the median nerve.

\section{Participants}

This review considers 2 distinct populations:

1. Asymptomatic adults (aged $\geq 16$ years) with no known pathology (past or present) affecting the peripheral nerves of the upper limb.

2. Adults with a diagnosis of carpal tunnel syndrome. For a study to be included, we did not impose any specific thresholds or criteria on the diagnosis of CTS, such as the presence of specific symptoms, provocative tests, aberrant electrophysiological parameters or imaging features.

\section{Search strategy}

The NICE Healthcare Databases (hdas.nice.org.uk) was searched according to Appendix 1 (Supplementary Materials) on 9th October 2020. The medRxiv and bioRxiv preprint archives were searched with the same strategy using the $\mathrm{R}$ package medrxivr ${ }^{13}$. Later, CitationChaser ${ }^{14}$ was used for forward and backward citation chasing based on the final list of included studies (eFigure 1).

\section{Study selection}

Three review authors (DR, JR and FR) independently screened titles and abstracts for relevance, in accordance with the eligibility criteria. The full texts of potentially eligible articles were obtained and 
again independently assessed by the same authors. Disagreements were resolved by discussion with RGW. The reasons for excluding studies are outlined in Appendix 2 (Supplementary Materials).

\section{Data extraction}

Three review authors (DR, JR and FR) independently double extracted all data. Thereafter, all datapoints were independently checked for accuracy by RGW. DTI parameters were extracted from the following anatomical levels of the median nerve: the distal radio-ulnar joint (DRUJ), the pisiform and the hook of the hamate, as these are three commonly used imaging landmarks which equate to the inlet, mid-point and outlet of the carpal tunnel. The nerve/hand was the unit of analysis. ${ }^{15}$ Many studies reported both the number of individuals and wrists scanned (as some studies involved bilateral scanning) but if not otherwise stated we assumed imaging was performed unilaterally. If data were missing, unclear or present in an unfavourable format then the authors were contacted by email with a request for more information. Four authors provided additional information upon request. ${ }^{16-19}$ When no reply was received, estimates were derived from graphs or imputed where possible. ${ }^{20}$

\section{Outcomes}

The primary outcome was to estimate the normal DTI metrics (FA and MD) of the median nerve in asymptomatic adults. The secondary outcomes were to estimate the differences in DTI metrics (FA and MD) between asymptomatic adults and patients with CTS, and explore the associations between DTI metrics and: age, echo time (TE), repetition time (TR), resolution, the number of diffusion sensitising gradient directions $\left(\mathrm{N}_{\mathrm{D}}\right)$ sampled per shell, the b-value(s), different methods of k-space sampling and inplane acceleration.

\section{Methodological quality assessment}

The risk of bias was independently assessed by three review authors (DR, JR and FR) using the ROBINS-I tool $^{21}$ and displayed graphically using robvis ${ }^{22}$. Disagreements were resolved by discussion with RGW.

\section{Statistical analysis}

The raw data are available via the Open Science Framework (https://osf.io/vqwkp/). The single study performed at 7 tesla $^{18}$ was excluded from all meta-analyses given its clinical disparity. Using the meta suite of Stata v16 (StataCorp, Texas), the mean FA and MD from asymptomatic adults were pooled to estimate the normative values. We performed direct comparisons meta-analysis of the mean differences in FA and MD between asymptomatic adults and patients with CTS. Meta-analyses were subgrouped by anatomical location. Restricted maximum likelihood was used to estimate the between-study variance $\left(\operatorname{tau}^{2}\right)$, with the Knapp and Hartung modification. Heterogeneity was quantified by $\mathrm{I}^{2} .{ }^{23}$ Using the metafor ${ }^{24}$ package, mixed-effects meta-regression was used to explore potential reasons for the observed heterogeneity in the direct comparisons meta-analysis of FA; the continuous covariates were age, in-plane resolution $\left(\mathrm{mm}^{2}\right)$, slice thickness $(\mathrm{mm})$, echo time (TE in $\left.\mathrm{ms}\right)$, b-value $\left(\mathrm{mm}^{2} / \mathrm{s}\right)$ and number of diffusionsensitising gradient directions $\left(\mathrm{N}_{\mathrm{D}}\right)$. TE and b-value were modelled as an interaction. Confidence intervals 
(Cl) were generated to the $95 \%$ level. To investigate the possibility of small-study effects for FA between asymptomatic adults and patients with CTS, a funnel plot was constructed with the pseudo Cls contoured by tau ${ }^{2} \cdot{ }^{24}$ EviAtlas was used to generate a map of the location of the 1 st author's institution. ${ }^{25}$

\section{Results}

Ultimately, 32 studies $^{16-19,26-53}$ were included (eFigure 2).

\section{Study characteristics}

Study characteristics are detailed in eTable 1. Overall, we included data from 2643 wrists belonging to 1575 asymptomatic adults and 1068 patients with CTS. Asymptomatic adults were a mean 6 years younger than patients with CTS $(95 \% \mathrm{Cl} 3,10)$. There were approximately twice as many females (1404:746) although this disparity was more pronounced in patients with CTS (660F:193M) than asymptomatic adults (737F:552M). The median number of authors was 6 (IQR 5-8) and studies were derived from 16 different countries (eFigure 3).

Ten studies (32\%) were performed at a field strength of $1 \cdot 5$ tesla $17,29,32,35,38,42,44,49,50,52$, twenty one (65\%) at 3 tesla $^{16,19,26-28,30,31,33,34,36,37,39-41,43,45-48,51,53}$, and one at 7 tesla $^{18}$. The median echo and repetition times were 87ms (IQR 65-91, range 21-103) and 7000ms (IQR 3800-7650, range 1470-10,254), respectively. Two studies used read-out segment echo-planar imaging (rsEPI) ${ }^{33,45}$, two did not specify the pulse sequence ${ }^{19,48}$ and the remainder used single-shot echo-planar imaging (ssEPI). Twelve studies described using the parallel imaging techniques (GRAPPA ${ }^{18,27,41}$, SENSE ${ }^{17,28,31,38,40,45,51}$ and CAIPIRINHA ${ }^{30}$ ) and six studies used partial Fourier transformations ${ }^{30,32,34,38,40,43,45}$. The median slice thickness was $3 \cdot 5 \mathrm{~mm}$ (IQR $2 \cdot 6-4 \cdot 0 \mathrm{~mm}$, range $1 \cdot 5-5 \mathrm{~mm}$ ). The median in-plane resolution was $1 \cdot 09 \mathrm{~mm}$ (IQR $0 \cdot 7-1 \cdot 5$, range $0 \cdot 4-1 \cdot 88$ ). Two studies investigated multiple b-values ${ }^{35,51}$ via discrete shells, although no studies reported whether acquisitions were half or whole shell and what sample scheme was used. The mean b-value was $1000 \mathrm{~s} / \mathrm{mm}^{2}$ (SD 270, range 325-2000). The median $\mathrm{N}_{\mathrm{D}}$ was 20 (IQR 15-25, range 6-32). A median of 3 signal averages (excitations) were obtained (IQR 2-5, range 1-12). When reported, the mean SNR of the b0 images was 25 (SD 12) 18,28,30,32,35,38.

The risk of bias for the included studies is summarised in eFigure 4. The majority of studies were at low risk of methodological bias.

\section{Evidence Synthesis: Asymptomatic Adults}

The FA of the median nerve in asymptomatic adults was reported in 29 studies $^{16-19,26-35,37-41,43-51,53}$. Overall, the normal FA was $0.58\left(95 \% \mathrm{Cl} 0.56,0.59 ; I^{2} 98 \%\right)$. The FA was highest at the level of the DRUJ (mean $0.61[95 \% \mathrm{Cl} 0.58,0.63] ; \mathrm{I}^{2} 96 \%$ ), dropping at the level of the pisiform to $0.57\left(95 \% \mathrm{Cl} 0.54,0.61 ; \mathrm{I}^{2}\right.$ $98 \%$ ) and lowest at the level of the hook of the hamate (mean 0.54 [95\% Cl 0.51, 0.57]; ${ }^{2}$ 95\%). 
The MD of the median nerve in asymptomatic adults was reported in 28 studies $^{16-19,26-35,37-41,43-51}$. Overall, the normal MD was $1 \cdot 138 \times 10^{-3} \mathrm{~mm}^{2} / \mathrm{s}\left(95 \% \mathrm{Cl} 1 \cdot 101,1 \cdot 174 ; I^{2} 99 \%\right)$. The MD was lowest at the level of the DRUJ (mean $1.073 \times 10^{-3} \mathrm{~mm}^{2} / \mathrm{s}$ [95\% Cl 1.019, 1.128]; $\mathrm{I}^{2} 93 \%$ ), increasing at the level of the pisiform (mean $1.180 \times 10^{-3} \mathrm{~mm}^{2} / \mathrm{s}[95 \% \mathrm{Cl} 1 \cdot 115,1 \cdot 244] ; \mathrm{I}^{2} 96 \%$ ) and highest at the level of the hook of the hamate (mean $1 \cdot 151 \times 10^{-3} \mathrm{~mm}^{2} / \mathrm{s}[95 \% \mathrm{Cl} 1 \cdot 086,1 \cdot 217] ; I^{2} 98 \%$ ).

\section{Evidence Synthesis: Patients with CTS}

The FA of the median nerve in patients with CTS was reported in 19 studies $^{16-19,26-28,31-33,37-39,44,46,48-}$ 50,52 . Overall, patients had a mean FA of 0.45 (95\% Cl 0.43, $0 \cdot 47 ; \mathrm{I}^{2}$ 95\%). The FA was lowest at the midpoint of carpal tunnel, at the level of the pisiform (mean 0.41 [95\% Cl 0.38, 0.43]; $I^{2} 86 \%$ ), compared to the levels of the DRUJ (mean 0.48 [95\% Cl 0.44, 0.52]; $\left.\right|^{2}$ 91\%) or hook of the hamate (mean $0.45[95 \% \mathrm{Cl}$ $\left.0 \cdot 42-0 \cdot 48] ; I^{2} 93 \%\right)$.

The MD of the median nerve in patients with CTS was reported in 18 studies $^{16-19,26-28,31-33,38,39,44,46,48-}$ 50,52 . Overall, patients with CTS had a pooled mean MD of $1.293 \times 10^{-3} \mathrm{~mm}^{2} / \mathrm{s}\left(95 \% \mathrm{Cl} 1 \cdot 227,1 \cdot 359 ; \mathrm{I}^{2}\right.$ $99 \%$ ). The MD was highest at the level of the pisiform (mean $1.372 \times 10^{-3} \mathrm{~mm}^{2} / \mathrm{s}[95 \% \mathrm{Cl} 1 \cdot 245-1 \cdot 500] ; I^{2}$ $98 \%), 1 \cdot 180 \times 10^{-3} \mathrm{~mm}^{2} / \mathrm{s}$ at the level of the DRUJ $\left(95 \% \mathrm{Cl} 1 \cdot 064,1 \cdot 295 ; \mathrm{I}^{2} 95 \%\right)$ and $1.335 \times 10^{-3} \mathrm{~mm}^{2} / \mathrm{s}$ at the level of the hook of the hamate $\left(95 \% \mathrm{Cl} 1 \cdot 259,1 \cdot 411 ; I^{2} 93 \%\right)$

\section{Direct Comparisons Meta-Analysis: Asymptomatic Adults vs. Patients with CTS}

Fourteen studies reported direct comparisons between asymptomatic adults and patients with CTS ${ }^{16-}$ $18,27,28,31-33,38,39,44,46,49,50$. All studies reported a lower FA in patients with CTS compared to asymptomatic adults (mean difference 0.09 [95\% $\mathrm{Cl} 0.07,0.11]$; Figure 2). The largest difference between asymptomatic adults and patients with CTS was at the mid-point of the carpal tunnel, at the level of the pisiform (mean difference $0 \cdot 12[95 \% \mathrm{Cl} 0 \cdot 09,0 \cdot 16]$ ).

Patients with CTS had a higher mean diffusivity than asymptomatic adults (mean difference $0.12 \mathrm{~mm}^{2} / \mathrm{s}$ $x 10^{-3}[95 \% \mathrm{Cl} 0 \cdot 08,0 \cdot 17]$, Figure 3 ). This difference was again most profound at the mid-point of the carpal tunnel, at the level of the pisiform (mean difference $0.16 \mathrm{~mm}^{2} / \mathrm{s} \times 10^{-3}[95 \% \mathrm{Cl} 0.05,0.27]$ ).

\section{Meta-regression}

Age was negatively associated with the FA in asymptomatic adults whereby each decade of life reduced the FA by approximately 0.003 (adjusted $\beta-2.79 \times 10^{-3}\left[95 \% \mathrm{Cl}-4.78 \times 10^{-3},-8.12 \times 10^{-4}\right] ; I^{2} 97 \%$ ). However, there was no relationship between age and FA in patients with CTS (adjusted $\beta 9.70 \times 10^{-4}[95 \% \mathrm{Cl}-2 \cdot 89$ $\left.x 10^{-3}, 4 \cdot 83 \times 10^{-3} ;\left.\right|^{2} 96 \%\right]$, Figure 4$)$. Increasing age was also associated with MD whereby each decade of life increased MD by approximately $0.108 \times 10^{-4} \mathrm{~mm}^{2} / \mathrm{s}\left(95 \% \mathrm{Cl} 0.073 \times 10^{-4}, 0.140 \times 10^{-4} ; \mathrm{I}^{2}\right.$ 99\%, Figure 5) with no significant difference between asymptomatic adults and patients with CTS. 
There was no relationship between $\mathrm{N}_{\mathrm{D}}$ and FA (eFigure 5) or MD (eFigure 6). The b-value was not associated with the FA (eFigure 7). There was an inverse relationship between the b-value and MD, whereby increments of $100 \mathrm{~mm}^{2} / \mathrm{s}$ reduced the diffusivity by $0.04 \times 10^{-3} \mathrm{~mm}^{2} / \mathrm{s}\left(\beta-3.849 \times 10^{-7} \mathrm{~mm}^{2} / \mathrm{s}\right.$ $\left[95 \% \mathrm{Cl}-5.019 \times 10^{-7},-2 \cdot 678 \times 10^{-7}\right] ; I^{2}$ 98\%, eFigure 8).

There were no significant differences between studies which used ssEPI or rsEPI. There was no association between the in-plane resolution (in square millimetres) and FA (eFigure 9) or MD (eFigure 10). Slice thickness was not associated with FA (eFigure 11) but was negatively associated with MD whereby increments of $1 \mathrm{~mm}$ reduced the MD by $6.023 \times 10^{-5} \mathrm{~mm}^{2} / \mathrm{s}\left(95 \% \mathrm{Cl} 9.754 \times 10^{-5}, 2.294 \times 10^{-5} ; \mathrm{I}^{2} 99 \%\right.$, eFigure 12)

The TE was not associated with FA or MD (eFigures 13 and 14). The TR was not associated with FA (eFigure 15) but longer repetition times were associated with lower estimates of MD, whereby increasing the TR by 1 second decreased the MD by $2.990 \times 10^{-6} \mathrm{~mm}^{2} / \mathrm{s}\left(95 \% \mathrm{Cl} 4.383 \times 10^{-6}, 1.598 \times 10^{-6}\right.$; eFigure 16).

Studies reporting the use of parallel imaging techniques (e.g. GRAPPA, SENSE or ASSET) yielded $5 \%$ higher estimates of FA ( $\beta 0.05$ [95\% Cl 0.02, 0.08]; $I^{2}$ 98\%, eFigure 17) when compared to studies which did not report this information. Parallel imaging methods were not associated with differences in the MD. There was insufficient data to explore different partial Fourier settings. There was no association between the number of signal averages and FA or MD (eFigure 18 and eFigure 19)

Ultimately, mixed-effects multivariable meta-regression showed that having CTS was the strongest independent moderator of the observed heterogeneity in FA (Table 1). Age explained some of the residual between-study variance. The experimental factors we modelled did not explain the residual heterogeneity.

There was no evidence of publication bias (Eggers $\beta 0 \cdot 10[95 \% \mathrm{Cl} 0 \cdot 06,0 \cdot 14] ; p=0 \cdot 134$, eFigure 20).

\section{Discussion}

This study demonstrates that throughout the length of the median nerve, patients with CTS have more isotropic diffusion than asymptomatic adults. The largest differences were observed at the mid-point of the carpal tunnel, at the level of pisiform, where patients with CTS had lower fractional anisotropy and higher mean diffusivity. Of clinical importance, we demonstrate that these real-world differences in independent of age and experimental (acquisition) conditions.

There are inherent problems with clinicians diagnosing CTS given that the available tests are largely unreliable. For example, nocturnal paraesthesias and many classical tests such as Phalen and Tinel, the scratch-collapse and sensory threshold testing have poor diagnostic value ${ }^{54,55}$. Despite the widespread use of neurophysiology studies in patients with suspected carpal tunnel syndrome, its diagnostic accuracy is also poor ${ }^{56}$. Moreover, surgeons still perform decompression surgery in the presence of 
normal tests ${ }^{57}$. It has been shown that DTI outperforms standard morphological imaging in patients with peripheral neuropathy ${ }^{34}$. Building on this premise and the poor performance of other tests, we demonstrate that DTI could fill a diagnostic void in the management of (at least unclear or complex) patients with CTS given that DTI generates reliable, reproducible and standardised proxy measures of nerve health. In the UK, the cost of a non-contrast MRI of the extremity is less than a neurophysiology exam ( $£ 389$ versus $£ 444 ; \$ 540$ versus $\$ 609) .58$ Therefore, we suggest that DTI could be a cheaper and potentially more accurate method of both diagnosing CTS and guiding treatment choices. Incorporating DTI in the real-world management of CTS would be difficult and require significant training for clinicians, changes to infrastructure and clinical pathways but ultimately, we show that DTI yields unique diagnostic information which could of significant and ubiquitous clinical value. Moreover, DTI could add value to the assessment of patients who don't improve after treatment or have bilateral symptomatology, where current tests (e.g., electrical studies) are inherently insensitive principally because they have no normal values.

We observed high statistical heterogeneity which has many potential explanations. The majority of the (statistical) heterogeneity was explained by the presence of CTS and it is plausible that the remainder is explained by the 'severity' of disease, which we were unable to capture. For example, we speculate that patients with more severe CTS (e.g., symptoms for years cases, resulting in profound demyelination, axonal loss and fibrosis) are likely to have lower FA and higher MD than patients with recent-onset mild CTS. Age also explained some of the observed heterogeneity and this is unsurprising, given that FA is known to fall in aging peripheral nerves ${ }^{59}$, just as it does in the white matter tracts of the brain ${ }^{60,61}$. This is because aging axons lose integrity, undergo demyelination and there is a simultaneous increase in extra-cellular fluid. Importantly, we showed that DTI metrics were sensitive to CTS after adjusting for age. Finally, in highly controlled and extreme conditions, user-specified factors ${ }^{62}$ such as the $\mathrm{SNR}^{63}, \mathrm{~b}$ value ${ }^{64,65}, N_{D}{ }^{66,67}$, distortion correction pipelines ${ }^{68,69}$, tensor fitting methods ${ }^{63}$ and partial volume effects ${ }^{70}$ have been shown affect the DTI parameter estimates, which may explain some of the remaining heterogeneity. Although we could not explore the effects of all these factors, in general we observed that experimental conditions had little or no significant effect on the measured FA or MD. Therefore, despite the statistical heterogeneity, DTI appears to be reliably sensitive to the microstructural changes of the median nerve which occur in CTS.

There were no significant associations between FA and MD, and several core elements of the pulse sequence. Therefore, we suggest that clinicians and researchers wishing to acquire data to simply fit a tensor could optimise their sequence as follows. As tensors are robust to varying b-values (in the hindered range) we suggest a b-value to $300-800 \mathrm{~mm}^{2} / \mathrm{s}$ because this enables a shorter TE, which improves SNR and mitigates the effects of T2 shine-through. By reducing the TE might enable users to take advantage of other vendor-specific options to improve data quality and reduce distortions. Given that increasing the $N_{D}$ has little effect on tensor fitting ${ }^{71}$, there are no crossing fibres to model and the median nerve is not tortuous (within or between voxels), we recommend limiting the $N_{D}$ to approximately 15 . The normal 
median nerve has a cross-sectional area of $9 \mathrm{~mm}^{2}(3.4 \mathrm{~mm} \text { diameter })^{72}$ and this increases with CTS ${ }^{73}$, so we recommend an in-plane resolution of approximately $1 \mathrm{~mm}^{2}$ to ensure that at least 1 voxel is not affected by partial voluming ${ }^{70}$ in all subjects. As that the median nerve is orthogonal to the imaging plane, the slice thickness can be tailored to achieve adequate SNR in the knowledge that it has little effect on the resultant FA. Until work is published to show the exact relaxation properties $\left(T 1, T 2, T 2^{\star}\right)$ of the median nerve we suggest that TR is minimised (e.g., 4500ms) to reduce scan time. When planning the number of signal averages, balanced full datasets should be acquired in opposing phase-encoding directions (blip-up, blip-down) to yield the best corrections of various distortions, via the FMRIB FSL pipeline ${ }^{74}$. It should be noted that thicker slices, longer repetition times and more signal averages are associated with lower estimates of MD.

\section{Limitations}

The main limitation of this study is the inherent and pervasive problem of CTS diagnosis which may have biased the findings. At present there is no internationally agreed diagnostic criteria for CTS and as such, there is clinical variation which is likely to be present in the includes studies. We planned to capture disease severity from the original studies, but this information was not available. As a matter of urgency, the community should work towards a consensus on objective criteria which constitute a diagnosis of CTS. Given the present absence of a reliable test for CTS, we speculate that DTI could fill the void and serve as the reference standard in future studies.

It is widely known that diffusion metrics in the brain are strongly dependent on the preprocessing distortion correction pipelines (i.e., software) used $^{74}$ but still there is no consensus on the minimum or indeed ideal suite of corrections to perform. This issue is compounded in the limb owing to an absence of research on the topic and hardware limitations. The majority of the included studies did not describe any form of distortion correction, how the diffusion data were reconstructed or how metrics were extracted from the median nerve. Before DTI can be used clinically, variations in these pipeline should be tested and a universal pipeline and standards for reporting diffusion data should be agreed by consensus.

Some readers will criticise our choice to pool estimates of FA and MD in the presence of high statistical heterogeneity. This was done because forest plots provide an important graphical representation of the variability of measurements in relation to experimental conditions (e.g., b-values and $N_{D}$ ) which are easy to interpret and moreover, meta-regression in this situation facilitates the exploration of heterogeneity.

\section{Conclusions}

We provide summary estimates of the normal FA and MD of the median nerve in asymptomatic adults. Furthermore, we show that diffusion throughout the length of the median nerve becomes more isotropic in patients with CTS.

\section{Declarations}




\section{Conflicts of interests}

None.

\section{Data sharing}

The raw data are available via the Open Science Framework (https://osf.io/vqwkp/). The statistical syntax is available from the senior author (RGW) upon request.

\section{Funding}

Ryckie Wade is a Doctoral Research Fellow funded by the National Institute for Health Research (NIHR, DRF-2018-11-ST2-028). The views expressed are those of the author(s) and not necessarily those of the United Kingdom's National Health Service, NIHR or Department of Health.

\section{Ethical approval}

Not applicable

\section{Contributions}

DR co-authored and registered the protocol, performed the searches, screened articles, extracted data, performed the risk of bias assessments and co-authored the manuscript. FR screened articles, extracted data, performed the risk of bias assessments and co-authored the manuscript. JR screened articles, extracted data, performed the risk of bias assessments and co-authored the manuscript. RGW conceived the study, co-authored the protocol, supervised the searches and citation chasing, checked all the extracted data for accuracy, performed the statistical analyses, created the artwork and co-authored the manuscript. All authors had full access to all the data in the study and had final responsibility for the decision to submit for publication.

\section{References}

1. Milone MT, Karim A, Klifto CS, et al. Analysis of Expected Costs of Carpal Tunnel Syndrome Treatment Strategies. HAND. 2019;14(3):317-323.

2. NEARY D, EAMES RA. THE PATHOLOGY OF ULNAR NERVE COMPRESSION IN MAN. Neuropathol. Appl. Neurobiol. 1975;1(1):69-88.

3. Pham K, Gupta R. Understanding the mechanisms of entrapment neuropathies. Neurosurg. Focus. 2009;26(2):E7.

4. Nath V, Schilling KG, Parvathaneni P, et al. Tractography reproducibility challenge with empirical data (TraCED): The 2017 ISMRM diffusion study group challenge. J. Magn. Reson. Imaging. 2020;51(1):234249. 
5. Vavasour IM, Meyers SM, Mädler B, et al. Multicenter Measurements of T 1 Relaxation and Diffusion Tensor Imaging: Intra and Intersite Reproducibility. J. Neuroimaging. 2019;29(1):42-51.

6. Prohl AK, Scherrer B, Tomas-Fernandez X, et al. Reproducibility of Structural and Diffusion Tensor Imaging in the TACERN Multi-Center Study. Front. Integr. Neurosci. 2019;13(July):1-15.

7. Kimura M, Yabuuchi H, Matsumoto R, et al. The reproducibility of measurements using a standardization phantom for the evaluation of fractional anisotropy (FA) derived from diffusion tensor imaging (DTI). Magn. Reson. Mater. Phys. Biol. Med. 2019:15-19.

8. Heckel A, Weiler M, Xia A, et al. Peripheral Nerve Diffusion Tensor Imaging: Assessment of Axon and Myelin Sheath Integrity. Plos One. 2015;10(6):e0130833.

9. Andersson G, Orädd G, Sultan F, et al. In vivo Diffusion Tensor Imaging, Diffusion Kurtosis Imaging, and Tractography of a Sciatic Nerve Injury Model in Rat at 9.4T. Sci. Rep. 2018;8(1):12911.

10. Friedrich P, Fraenz C, Schlüter C, et al. The Relationship between Axon Density, Myelination, and Fractional Anisotropy in the Human Corpus Callosum. Cereb. Cortex. 2020;30(4):2042-2056.

11. Higgins JPT, Green S (editors). Cochrane Handbook for Systematic Reviews of Interventions Version 5.1.0 [updated March 2011]. Cochrane Collab. 2011.

12. Page MJ, McKenzie JE, Bossuyt PM, et al. The PRISMA 2020 statement: an updated guideline for reporting systematic reviews. Syst. Rev. 2021;10(1).

13. McGuinness L, Schmidt L. medrxivr: Accessing and searching medRxiv and bioRxiv preprint data in R. J. Open Source Softw. 2020;5(54):2651.

14. Haddaway NR. citationchaser: an R package for forward and backward citations chasing in academic searching. Zenodo; 2021. Available at: https://zenodo.org/record/4533747. Accessed February 15, 2021.

15. Wade RG, Takwoingi Y, Wormald JCR, et al. MRI for Detecting Root Avulsions in Traumatic Adult Brachial Plexus Injuries: A Systematic Review and Meta-Analysis of Diagnostic Accuracy. Radiology. 2019;293(1):125-133.

16. Stein D, Neufeld A, Pasternak O, et al. Diffusion tensor imaging of the median nerve in healthy and carpal tunnel syndrome subjects. J. Magn. Reson. Imaging. 2009;29(3):657-662.

17. Razek AAKA, Shabana AAE, El Saied TO, et al. Diffusion tensor imaging of mild-moderate carpal tunnel syndrome: correlation with nerve conduction study and clinical tests. Clin. Rheumatol. 2017;36(10):2319-2324.

18. Schmid AB, Campbell J, Hurley SA, et al. Feasibility of Diffusion Tensor and Morphologic Imaging of Peripheral Nerves at Ultra-High Field Strength: Invest. Radiol. 2018;53(12):705-713. 
19. Paniandi V, George J, Goh KJ, et al. MR neurography of median nerve using diffusion tensor. Neurol. Asia. 2018:9.

20. Wan X, Wang W, Liu J, et al. Estimating the sample mean and standard deviation from the sample size, median, range and/or interquartile range. BMC Med. Res. Methodol. 2014;14(1):135.

21. Sterne JA, Hernán MA, Reeves BC, et al. ROBINS-l: a tool for assessing risk of bias in non-randomised studies of interventions. BMJ. 2016;355:i4919.

22. McGuinness LA, Higgins JPT. Risk-of-bias VISualization (robvis): An R package and Shiny web app for visualizing risk-of-bias assessments. Res. Synth. Methods. 2020;(February):jrsm.1411.

23. Higgins JPT, Thompson SG. Quantifying heterogeneity in a meta-analysis. Stat. Med. 2002;21(11):1539-1558.

24. Viechtbauer W. Conducting Meta-Analyses in R with the metafor Package. J. Stat. Softw. 2010;36(3).

25. Haddaway NR, Feierman A, Grainger MJ, et al. EviAtlas: a tool for visualising evidence synthesis databases. Environ. Evid. 2019;8(1):22.

26. Barcelo $C$, Faruch M, Lapègue $F$, et al. 3-T MRI with diffusion tensor imaging and tractography of the median nerve. Eur Radiol.:7.

27. Brienza M, Pujia F, Colaiacomo MC, et al. 3T diffusion tensor imaging and electroneurography of peripheral nerve: A morphofunctional analysis in carpal tunnel syndrome. J. Neuroradiol. 2014;41(2):124-130.

28. Koh SH, Kwon BC, Park C, et al. A comparison of the performance of anatomical MRI and DTI in diagnosing carpal tunnel syndrome. Eur. J. Radiol. 2014;83(11):2065-2073.

29. Edward R, Abdelalim AM, Ashour AS, et al. A study of diffusion tensor imaging of median nerve in diabetic peripheral neuropathy. Egypt. J. Neurol. Psychiatry Neurosurg. 2020;56(1).

30. Filli L, Piccirelli M, Kenkel D, et al. Accelerated magnetic resonance diffusion tensor imaging of the median nerve using simultaneous multi-slice echo planar imaging with blipped CAIPIRINHA. Eur. Radiol. 2016;26(6):1921-1928.

31. Guggenberger R, Markovic D, Eppenberger P, et al. Assessment of Median Nerve with MR Neurography by Using Diffusion-Tensor Imaging: Normative and Pathologic Diffusion Values. Radiology. 2012;265(1):194-203.

32. Wang I-Ming; Huang, Han-Wei; Chen, Pei-Yin; Tsai, Hong-Ming; Liu, Yi Sheng; Lin, Chou-Ching K. CKJ. Carpal tunnel syndrome assessed with diffusion tensor imaging: comparison with electrophysiological studies of patients and healthy volunteers. 2012;81(11):3378-3383. 
33. Klauser AS, Abd Ellah M, Kremser C, et al. Carpal tunnel syndrome assessment with diffusion tensor imaging: Value of fractional anisotropy and apparent diffusion coefficient. Eur. Radiol. 2017:1-7.

34. Breckwoldt MO, Stock C, Xia A, et al. Diffusion Tensor Imaging Adds Diagnostic Accuracy in Magnetic Resonance Neurography. Invest. Radiol. 2015;50(8):7.

35. Andreisek Lawrence M.; Kassner, Andrea; Tomlinson, George; Sussman, Marshall S. GW. Diffusion tensor imaging and fiber tractography of the median nerve at 1.5T: optimization of $b$ value. 2008;38(1):51-59.

36. Hiltunen J, Suortti T, Arvela S, et al. Diffusion tensor imaging and tractography of distal peripheral nerves at 3 T. Clin. Neurophysiol. 2005;116(10):2315-2323.

37. Kabakci N, Gürses B, Firat Z, et al. Diffusion tensor imaging and tractography of median nerve: Normative diffusion values. Am. J. Roentgenol. 2007;189(4):923-927.

38. Khalil C, Hancart C, V LT, et al. Diffusion tensor imaging and tractography of the median nerve in carpal tunnel syndrome: preliminary results. Eur. Radiol. 2008;18(10):2283-2291.

39. Tasdelen N, Gurses B, Kilickesmez O, et al. Diffusion tensor imaging in carpal tunnel syndrome. Diagn. Interv. Radiol. 2011:60-66.

40. Zhou Y, Kumaravel M, Patel VS, et al. Diffusion tensor imaging of forearm nerves in humans. J. Magn. Reson. Imaging. 2012;36(4):920-927.

41. Guggenberger R, Nanz D, Bussmann L, et al. Diffusion tensor imaging of the median nerve at 3.0T using different MR scanners: Agreement of FA and ADC measurements. Eur. J. Radiol. 2013;82(10):e590-e596.

42. Naraghi A, da Gama Lobo L, Menezes R, et al. Diffusion tensor imaging of the median nerve before and after carpal tunnel release in patients with carpal tunnel syndrome: feasibility study. Skeletal Radiol. 2013;42(10):1403-1412.

43. Lindberg Antoine; Le Viet, Dominique; Maier, Marc A.; Drapé, Jean-Luc PG; F. Diffusion tensor imaging of the median nerve in recurrent carpal tunnel syndrome - initial experience. 2013;23(11):3115-3123.

44. Cingoz Sedat Giray; Alis, Deniz; Samanci, Cesur; Kandemirli, Guzin Cakir; Adatepe, Nurten Uzun MK. Evaluation of median nerve by shear wave elastography and diffusion tensor imaging in carpal tunnel syndrome. 2018;101(NA):59-64.

45. Yao L, Gai N. Median nerve cross-sectional area and MRI diffusion characteristics: Normative values at the carpal tunnel. Skeletal Radiol. 2009;38(4):355-361. 
46. Kwon BC, Koh SH, Hwang SY. Optimal Parameters and Location for Diffusion-Tensor Imaging in the Diagnosis of Carpal Tunnel Syndrome: A Prospective Matched Case-Control Study. Am. J. Roentgenol. 2015;204(6):1248-1254.

47. Heckel A, Weiler M, Xia A, et al. Peripheral Nerve Diffusion Tensor Imaging: Assessment of Axon and Myelin Sheath Integrity. Yang J, ed. PLOS ONE. 2015;10(6):e0130833.

48. Hiltunen Erika; Numminen, Jussi; Lindfors, Nina; Göransson, Harry; Hari, Riitta JK. Pre- and postoperative diffusion tensor imaging of the median nerve in carpal tunnel syndrome. 2012;22(6):13101319.

49. Wafaie AM, Afifi LM, Moussa KM, et al. Role of diffusion tensor imaging in carpal tunnel syndrome: $A$ case control comparative study to electrophysiological tests and clinical assessment. Egypt. J. Radiol. Nucl. Med. 2018;49(4):1068-1075.

50. Bulut HT, Yildirim A, Ekmekci B, et al. The Diagnostic and Grading Value of Diffusion Tensor Imaging in Patients with Carpal Tunnel Syndrome. Acad. Radiol. 2014;21(6):767-773.

51. Ding WQ, Zhou XJ, Tang JB, et al. Three-dimensional display of peripheral nerves in the wrist region based on MR diffusion tensor imaging and maximum intensity projection post-processing. Eur. J. Radiol. 2015;84(6):1116-1127.

52. Yildirim Haci Taner; Ekmekçi, Burcu; Sürücü, Gülseren Dost; Karabiber, Mehmet AB. Use of diffusion tensor imaging for nonsurgical treatments of carpal tunnel syndrome. 2014;50(6):950-955.

53. Pridmore MD, Glassman GE, Pollins AC, et al. Initial findings in traumatic peripheral nerve injury and repair with diffusion tensor imaging. Ann. Clin. Transl. Neurol. 2021;8(2):332-347.

54. Simon J, Lutsky K, Maltenfort M, et al. The Accuracy of the Scratch Collapse Test Performed by Blinded Examiners on Patients With Suspected Carpal Tunnel Syndrome Assessed by Electrodiagnostic Studies. J. Hand Surg. 2017:1-5.

55. D’Arcy CA. Does This Patient Have Carpal Tunnel Syndrome? JAMA. 2000;283(23):3110.

56. Lu Y-T, Deol AK, Sears ED. The Association Between Electrodiagnostic Severity and Treatment Recommendations for Carpal Tunnel Syndrome. J. Hand Surg. 2021;46(2):92-98.

57. Mackenzie SP, Stone OD, Jenkins PJ, et al. Carpal tunnel decompression in patients with normal nerve conduction studies. J. Hand Surg. Eur. Vol. 2020;45(3):260-264.

58. Anon. NIHR 2020 Investigation and Intervention Tariff 1.2.pdf.

59. Kronlage M, Schwehr V, Schwarz D, et al. Peripheral nerve diffusion tensor imaging (DTI): normal values and demographic determinants in a cohort of 60 healthy individuals. Eur. Radiol. 
2018;28(5):1801-1808.

60. Yap QJ, Teh I, Fusar-Poli P, et al. Tracking cerebral white matter changes across the lifespan: insights from diffusion tensor imaging studies. J. Neural Transm. 2013;120(9):1369-1395.

61. Watanabe M, Sakai O, Ozonoff A, et al. Age-related Apparent Diffusion Coefficient Changes in the Normal Brain. Radiology. 2013;266(2):575-582.

62. Helmer KG, Chou M-C, Preciado RI, et al. Multi-site study of diffusion metric variability: effects of site, vendor, field strength, and echo time on regions-of-interest and histogram-bin analyses. In: Gimi B, Krol A, eds. Physiology \& Behavior.Vol 176.; 2016:97882U.

63. Jones DK, Basser PJ. "Squashing peanuts and smashing pumpkins": How noise distorts diffusionweighted MR data. Magn. Reson. Med. 2004;52(5):979-993.

64. Schilling KG, Nath V, Blaber J, et al. Effects of b-value and number of gradient directions on diffusion MRI measures obtained with Q-ball imaging. Styner MA, Angelini ED, eds. Proc SPIE Int Soc Opt Eng. 2017:101330N.

65. Qin W, Shui Yu C, Zhang F, et al. Effects of echo time on diffusion quantification of brain white matter at 1.5T and 3.0T. Magn. Reson. Med. 2009;61(4):755-760.

66. Haakma W, Pedersen M, Froeling M, et al. Diffusion tensor imaging of peripheral nerves in non-fixed post-mortem subjects. Forensic Sci. Int. 2016;263:139-146.

67. Giannelli M, Cosottini M, Michelassi MC, et al. Dependence of brain DTI maps of fractional anisotropy and mean diffusivity on the number of diffusion weighting directions. J. Appl. Clin. Med. Phys. 2010;11(1):176-190.

68. Taylor PA, Alhamud A, van der Kouwe A, et al. Assessing the performance of different DTI motion correction strategies in the presence of EPI distortion correction. Hum. Brain Mapp. 2016;37(12):44054424.

69. Haddad SMH, Scott CJM, Ozzoude M, et al. Comparison of quality control methods for automated diffusion tensor imaging analysis pipelines. Yap P-T, ed. PLOS ONE. 2019;14(12):e0226715.

70. Vos SB, Jones DK, Viergever MA, et al. Partial volume effect as a hidden covariate in DTI analyses. Neurolmage. 2011;55(4):1566-1576.

71. Ni H, Kavcic V, Zhu T, et al. Effects of number of diffusion gradient directions on derived diffusion tensor imaging indices in human brain. AJNR Am. J. Neuroradiol. 2006;27(8):1776-1781.

72. Klauser Ethan J.; De Zordo, Tobias; Feuchtner, Gudrun; Arora, Rohit; Gruber, Johann; Martinoli, Carlo; Löscher, Wolfgang AH. Carpal Tunnel Syndrome Assessment with US: Value of Additional Cross-sectional 
Area Measurements of the Median Nerve in Patients versus Healthy Volunteers. 2008;250(1):171-177.

73. Roomizadeh P, Eftekharsadat B, Abedini A, et al. Ultrasonographic Assessment of Carpal Tunnel Syndrome Severity: A Systematic Review and Meta-Analysis. Am. J. Phys. Med. Rehabil. 2019;98(5):373381.

74. Brun L, Pron A, Sein J, et al. Diffusion MRI: Assessment of the Impact of Acquisition and Preprocessing Methods Using the BrainVISA-Diffuse Toolbox. Front. Neurosci. 2019;13:536.

\section{Tables}

Table 1. Mixed-effects meta-regression

\begin{tabular}{|c|c|c|c|}
\hline Covariates & $\begin{array}{l}\text { Adjusted Change in } \\
\text { Fractional Anisotropy ( } \beta \text { ) }\end{array}$ & $95 \% \mathrm{Cl}$ & $\begin{array}{l}\text { Resampled } \\
\text { p-value }\end{array}$ \\
\hline $\begin{array}{l}\text { Patients with Carpal Tunnel } \\
\text { Syndrome }\end{array}$ & $-8.57 \times 10^{-2}$ & $-0 \cdot 13-0 \cdot 06$ & $0 \cdot 000$ \\
\hline Age in Years & $-2.92 \times 10^{-3}$ & $\begin{array}{l}-4.54 \times 10^{-3} \\
-7 \cdot 34 \times 10^{-4}\end{array}$ & 0.007 \\
\hline Repetition Time (ms) & $-8.98 \times 10^{-6}$ & $\begin{array}{l}-1 \cdot 14 \times 10^{-5} \\
6 \cdot 77 \times 10^{-8}\end{array}$ & $0 \cdot 053$ \\
\hline $\begin{array}{l}\text { Number of diffusion sensitising } \\
\text { gradient directions }\end{array}$ & $2.27 \times 10^{-3}$ & $\begin{array}{l}-8.39 \times 10^{-4} \\
2.99 \times 10^{-3}\end{array}$ & $0 \cdot 271$ \\
\hline Echo Time $(\mathrm{ms}) \&$ b-value $\left(\mathrm{mm}^{2} / \mathrm{s}\right)^{*}$ & $-1.51 \times 10^{-8}$ & $\begin{array}{l}-5 \cdot 27 \times 10^{-7} \\
4.86 \times 10^{-7}\end{array}$ & 0.936 \\
\hline \multicolumn{4}{|l|}{ Adjusted $R^{2}=46 \%, \operatorname{tau}^{2}=0.0047, P^{2}=97 \%$} \\
\hline \multicolumn{4}{|c|}{$\begin{array}{l}\text { *Echo time is a function of the } b \text {-value as larger } b \text {-values mandate relatively longer echo times, so } \\
\text { these variables are modelled as the product to minimise the number of covariables, mitigate } \\
\text { collinearity and model the interaction between the two variables }\end{array}$} \\
\hline
\end{tabular}

\section{Figures}



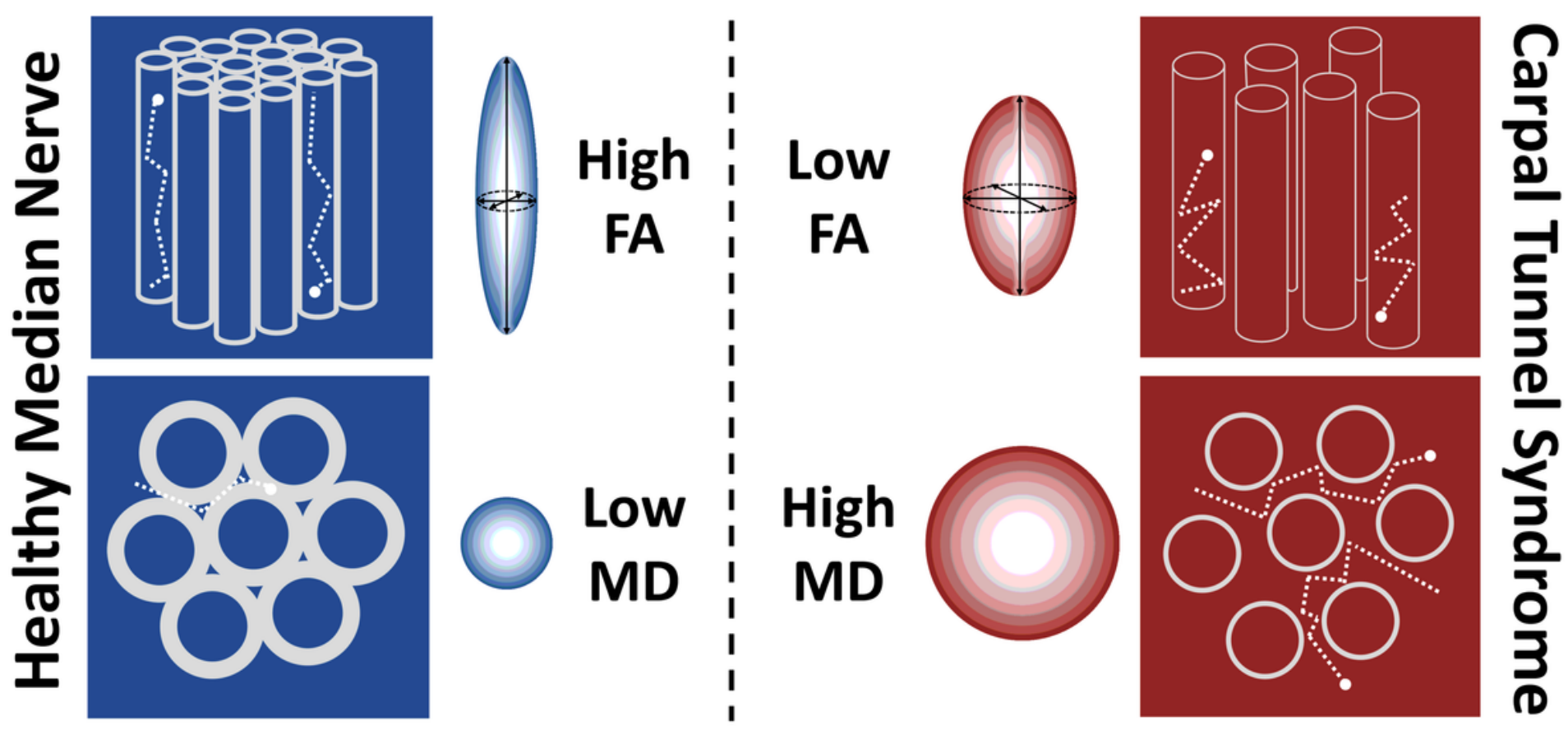

Figure 1

A diagram of nerve fibres (top) and in cross-section (bottom) demonstrating how diffusion tensor imaging metrics change in CTS. In healthy nerves, the axons are enveloped by thick myelin sheaths and arranged relatively tightly, which restricting the diffusion of water to the long axis of the nerve. Chronic compression leads to distortion of the axonal architecture, demyelination and as such, degradation of physiological barriers to the diffusion of water diffusion. Consequently, more diffusion occurs perpendicular to the long axis of the nerve and water is more free to diffuse around the fibres, reducing the factional anisotropy (FA) and increasing the magnitude of diffusion (mean diffusivity, MD). 


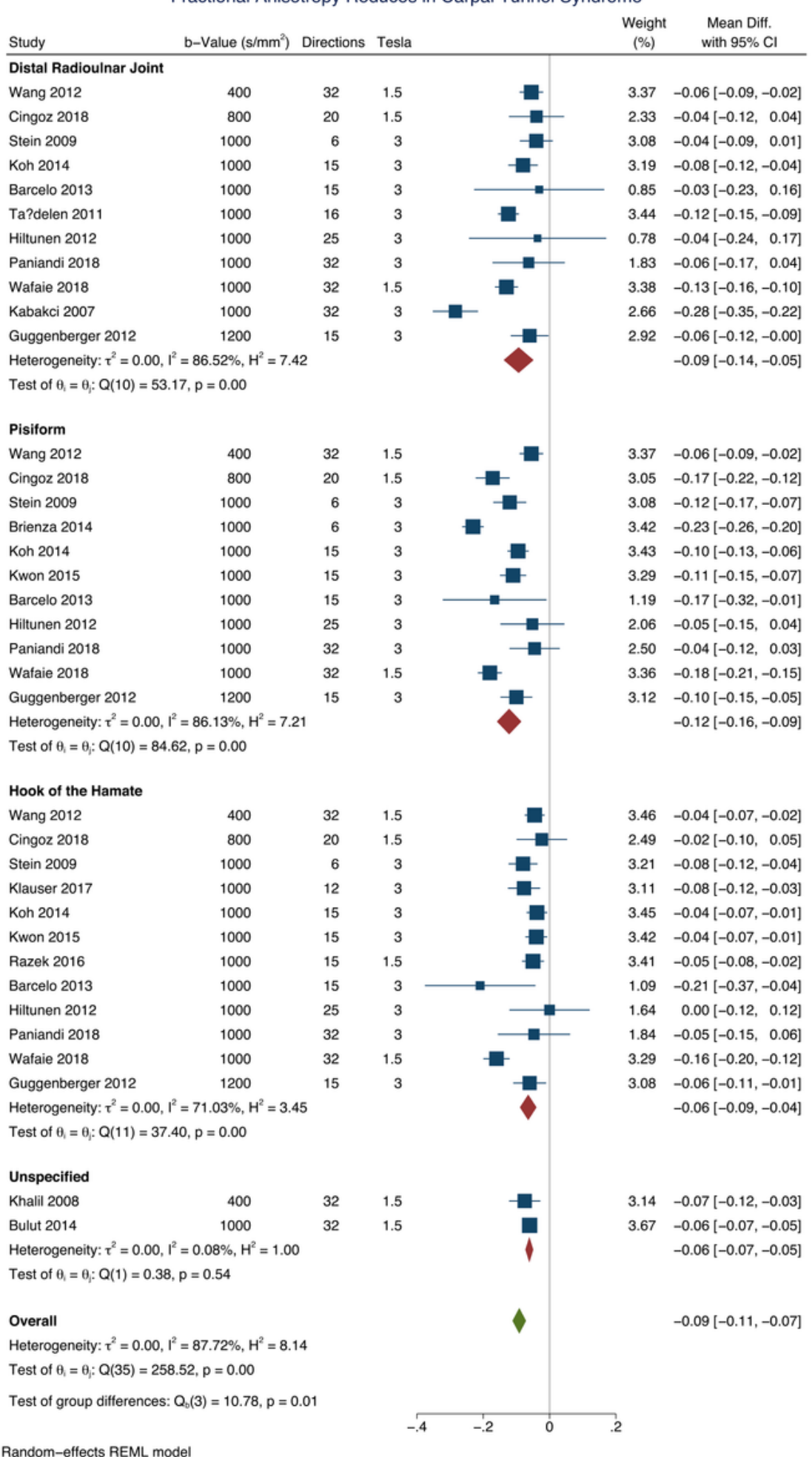

Sorted by: bval directions

Figure 2

A forest plot of the fractional anisotropy of the median nerve, at 3 anatomical levels, between asymptomatic adults and patients with carpal tunnel syndrome 


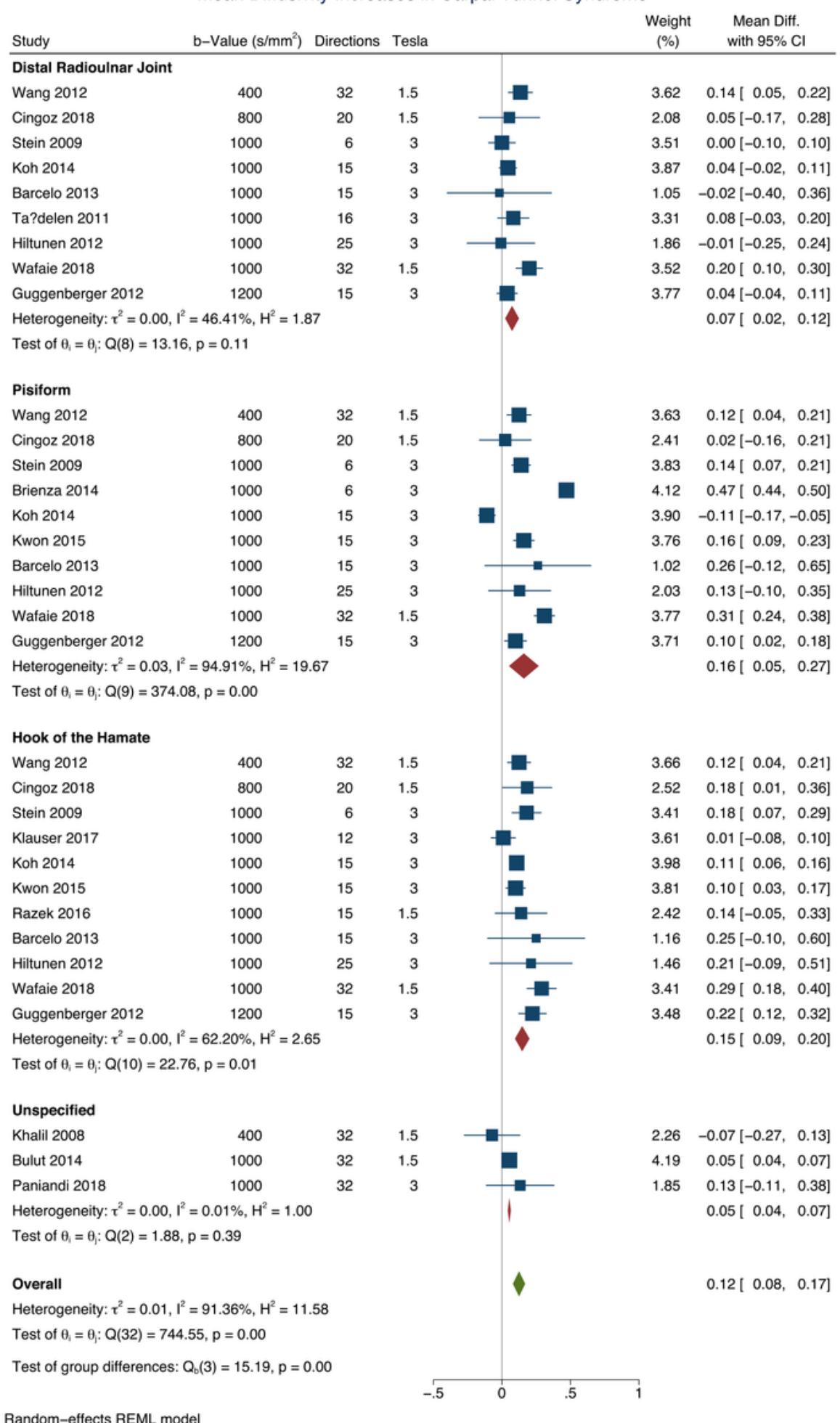

Sorted by: bval directions

Figure 3

A forest plot of the mean diffusivity of the median nerve, at 3 anatomical levels, between asymptomatic adults and patients with carpal tunnel syndrome 


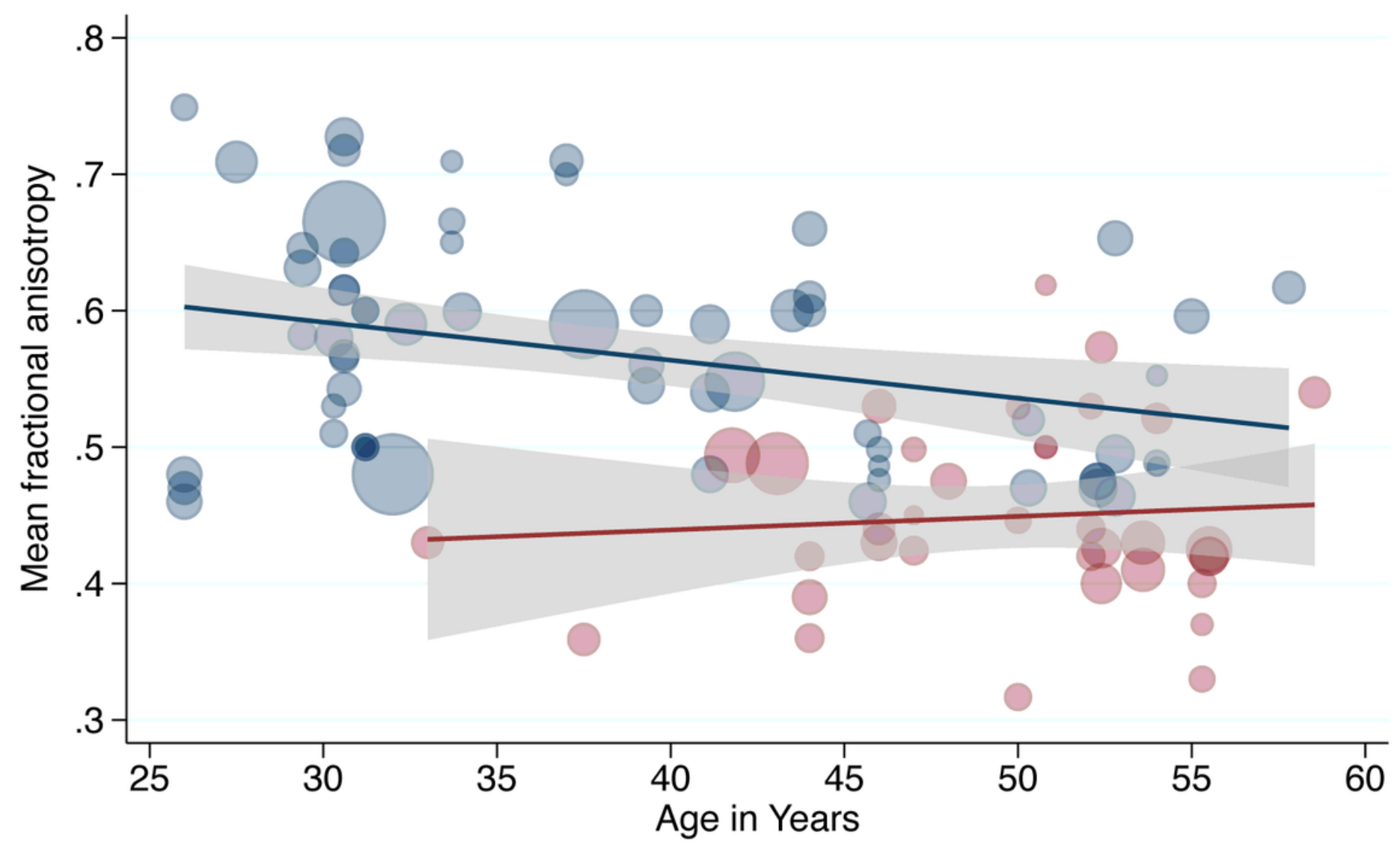

- Asymptomatic Volunteers Linear fit

- Patients with CTS L Linear fit

Figure 4

A scatterplot of study-level estimates of fractional anisotropy in asymptomatic adults and patients with carpal tunnel syndrome, against age in years. The size of the points corresponds to the precision (inverse variance) of the study. 


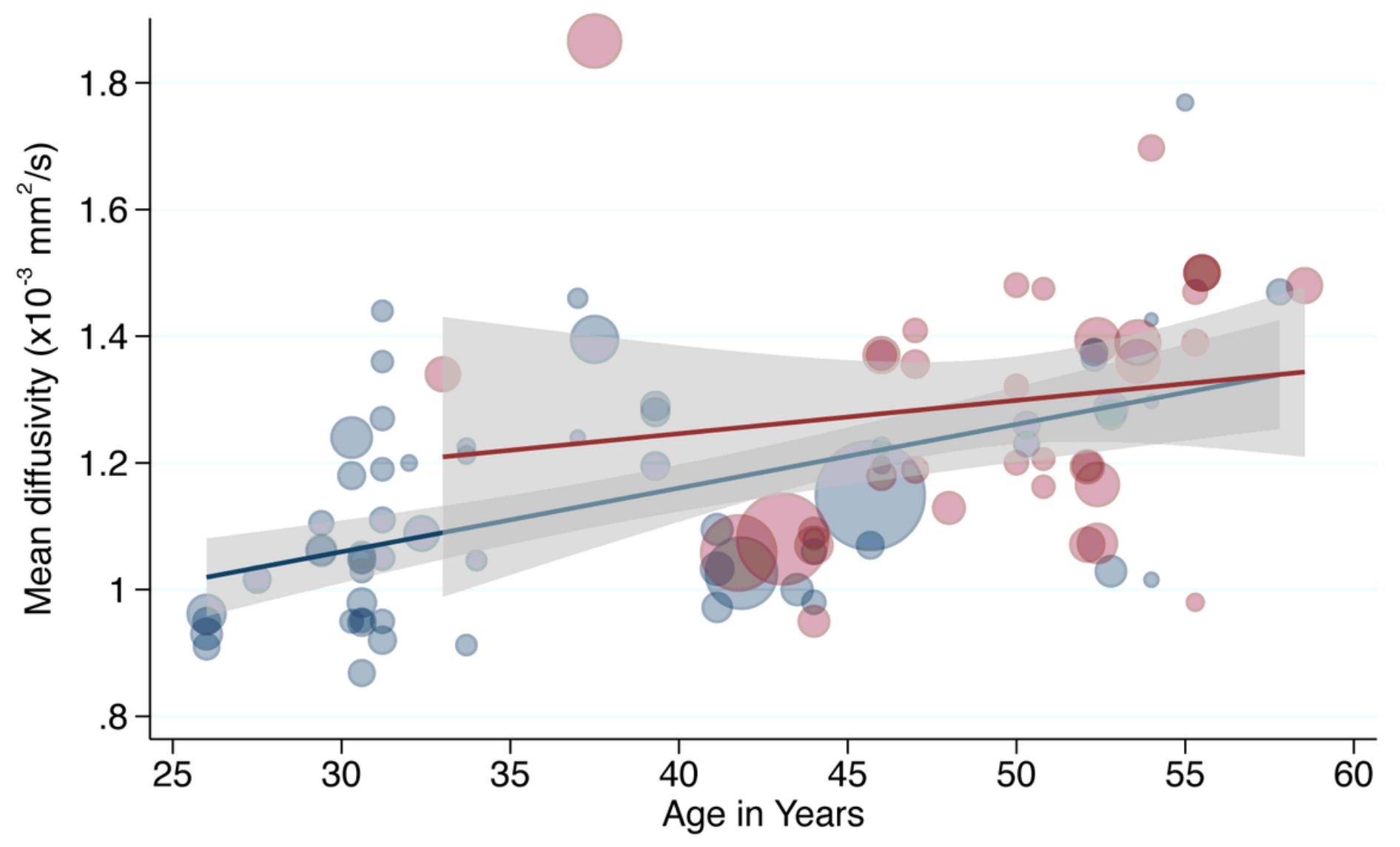

$\begin{array}{lll}\text { - Asymptomatic Volunteers } & \text { Linear fit } & 95 \% \mathrm{Cl} \\ \text { - Patients with CTS } & \text { Linear fit } & \end{array}$

Figure 5

A scatterplot of study-level estimates of mean diffusivity in asymptomatic adults and patients with carpal tunnel syndrome, against age in years. The size of the points corresponds to the precision (inverse variance) of the study.

\section{Supplementary Files}

This is a list of supplementary files associated with this preprint. Click to download.

- SupplementaryMaterials.docx 